\title{
Asociación entre enfermedad metabólica y severidad de aterosclerosis coronaria evaluada por Score de Gensini
}

\author{
Benjamín Stockins ${ }^{1,2}$, Luis Quiñiniir ${ }^{1}$, Virginia Iturrieta ${ }^{1}$, Gabriel López ${ }^{1}$, Hugo Hernández ${ }^{a}$, Rocío \\ Serpella, Luis Bustos ${ }^{b}$ \\ 1.- Departamento de Medicina Interna, Universidad de la Frontera \\ 2.- Centro Cardiovascular, Hospital Dr. Hernán Henríquez Aravena, de Temuco \\ a.- Interno Medicina Universidad de La Frontera \\ b.- Estadístico Universidad de la Frontera, CIGES
}

Introducción: La asociación entre cardiopatía coronaria (CC) y estados de disglicemia está bien establecida. Sin embargo, aún cuando la diabetes mellitus (DM) corresponde a uno de los principales factores de riesgo para $\mathrm{CC}$, la asociación con el síndrome metabólico (SM) parece menos clara. Nuestro objetivo fue evaluar el grado de compromiso aterosclerótico del árbol coronario de acuerdo a la existencia de SM y DM.

Pacientes y método: Se analizó los datos de 413 pacientes sometidos a coronariografía electiva en el período de un año. Se dividió la muestra en 3 grupos: pacientes diabéticos, pacientes con SM (no diabéticos) y pacientes sin enfermedad metabólica (no SM, no DM). Se consideró como portador de CC a todo paciente con estenosis $>50 \%$ en cualquier arteria coronaria, la severidad de la enfermedad coronaria fue medida por el Score de Gensini. Se utilizó análisis de varianza (Barlett) con comparación múl- tiple de Scheffe y prueba no paramétrica de Kruskal-Wallis en aquellos grupos con varianzas distintas. El protocolo fue aprobado por el Comité Ético Científico del Servicio de Salud Araucanía Sur.

Resultados: La edad promedio fue $63.8 \pm 11,17$ años. La prevalencia de SM fue 38\% ( $\mathrm{n}=158)$, DM $45 \%(\mathrm{n}=186)$ y no tenían alteraciones metabólicas $17 \%$ de los pacientes $(n=69)$. La prevalencia global de enfermedad coronaria fue $52,7 \%$. La prevalencia en los distintos grupos fue $33.9 \%$ en aquellos sin enfermedad metabólica, $52.2 \%$ en los portadores de SM y $62.2 \%$ en los pacientes con DM ( $p=0.001)$. En cuanto a la severidad de la CC, el score de Gensini fue $18,22,04$ y 29,6 respectivamente $(\mathrm{p}=0,04)$.

Conclusiones: Este estudio observacional sugiere que, comparados con sujetos sin enfermedad metabólica tanto la prevalencia de CC, como su severidad son mayores en pacientes con SM aislado y mayores aún en portadores de DM.

\section{Correspondencia:}

Dr. Benjamín Stockins Fernández

Teléfono 53722607

benjamín.stockins@ufrontera.cl 


\section{Association between metabolic disease and the severity of coronary artery disease measured by Gensini Score}

Introduction: The association between coronary artery (CAD) and dysglicemia is well established. Diabetes (DM) is recognized as one of the most important coronary risk factors, but the association of CAD and metabolic syndrome (MS) is less well defined. Our objective was to evaluate the degree of coronary atherosclerotic disease in relation to the presence of MS and DM .

Patients and Method: The data of 413 patients electively studied with coronary angiography over a period of one year was analyzed. Patients were divided into three groups: Patients with DM, patients with MS (without DM) and patients without a metabolic disorder (no DM, no MS). The severity of CAD was measured by the Gensini Score.
Results: Mean age was $63,8 \pm 11,17$ years. The prevalence of patients with MS was $38 \%(\mathrm{n}=158)$, with DM 45\% ( $\mathrm{n}=186)$ and without a metabolic disorder $17 \%(n=69)$. The global prevalence of CAD was $52,7 \%$. The prevalence of CAD in the different groups was as follows: Absence of metabolic disease 33.9\%, patients with MS 52.2\% and patients with DM 62.2\% $(\mathrm{p}<0.001)$.

Conclusions: This observational study suggests that, compared to subjects without metabolic disease, the prevalence and severity of CAD are higher in patients with MS and even higher in patients with DM.

Keywords: Diabetes, Metabolic Syndrome, Coronary artery disease, Coronary stenosis. 


\section{Introducción:}

Las enfermedades del sistema circulatorio son la primera causa de mortalidad en nuestro país, correspondiendo al $27 \%$ de las causas de muerte ${ }^{1}$. Dentro de los factores de riesgo más consistentes se encuentran las alteraciones del metabolismo glicémico ${ }^{2}$ y se sabe que la resistencia a insulina induce la ateroesclerosis incluso antes de desarrollarse una diabetes mellitus (DM) franca ${ }^{3}$.

La DM constituye uno de los factores de riesgo cardiovascular más importante, ${ }^{4}$ llegando a ser considerado equivalente de riesgo en cualquier localización de enfermedad aterosclerótica ${ }^{5}$. Asimismo, la cardiopatía coronaria (CC) es más prevalente, de peor pronóstico y la causa más frecuente de muerte en pacientes diabéticos. ${ }^{6}$ En estos pacientes existe mayor prevalencia de isquemia silente cuando son estudiados con tests funcionales como ecocardiografía stress o cintigrafía $\mathrm{MIBI}^{7}$.

El riesgo de presentar eventos coronarios aumenta progresivamente, incluso antes de desarrollarse DM. En el estudio prospectivo Nurses Health (NHS) el 5\% de las mujeres inicialmente no diabéticas desarrollaron DM a 20 años de seguimiento. Estas pacientes presentaron un riesgo relativo ajustado de 3.17 (95\% CI 2.61 a 3.85) para presentar eventos coronarios ${ }^{8}$.

Por otro lado, el Síndrome Metabólico (SM) se ha identificado desde 1988 como la presencia de intolerancia a la glucosa con hiperinsulinemia, asociada a hipertrigliceridemia, bajas concentraciones de CHDL, hipertensión arterial (HA) y obesidad central. ${ }^{9}$ Diversos metanálisis demuestran que el SM aumenta el riesgo de muerte cardiovascular ${ }^{10-11-12-13}$ y por otro lado se ha reportado una prevalencia de enfermedad coronaria de $51 \%$ en estos pacientes ${ }^{14}$. Sin embargo también existen publicaciones que no muestran mayor riesgo cardiovascular del SM que la suma de sus partes ${ }^{15}$, con lo cual se ha criticado su utilidad como diagnóstico clínico ${ }^{16}$. Pese a esto, la Organización Mundial de Salud (OMS) ${ }^{17}$, American Heart Association, National Cholesterol Education Program Adult Treatment Panel III (NCEP ATPIII) ${ }^{5}$ y la Federación Internacional de Diabetes (IDF) ${ }^{18}$, aceptaron al SM como un diagnóstico útil.

La Encuesta Nacional de Salud 2009-10, utilizando la definición ATPIII Update, con puntos de corte de cintura validados para Chile, reportó una prevalencia de SM de $35,3 \%$ para adultos, con una mayor prevalencia en la Región de La Araucanía (42\%). Existen diferencias entre hombres y mujeres $(41,6 \% \%$ y $30,9 \%$, respectivamente) y a los 65 años la prevalencia sube al 51,6\%. Así mismo, la prevalencia de DM en nuestro país corresponde a un $9 \%$, siendo en la La Araucanía de 12,7\% ${ }^{19}$.
El presente trabajo tiene por objetivo conocer el impacto que tiene la DM y el SM sobre la severidad de la CC, utilizando el puntaje de Gensini como evaluación angiográfica.

\section{Material y Método}

Pacientes: Se realizó un estudio descriptivo, que incluyó a 583 pacientes $(100 \%)$ que se sometieron a coronariografía en el Hospital Dr. Hernán Henríquez Aravena, de Temuco, entre el 15 de junio 2011 y el 5 junio 2012. Los pacientes fueron estudiados con coronariografía electiva dada la existencia de síndrome anginoso, estudio de arritmias malignas, estudio preoperatorio y otros. Se analizaron variables clínicas y de laboratorio incluyendo perfil lipídico, glicemia de ayuno, hemoglobina A1C y creatininemia.

Pacientes con Síndrome Coronario Agudo (SCA), en los cuales los niveles de glicemia y lípidos pudieran estar alterados por esta situación, fueron excluidos. Igualmente, se excluyó a pacientes con antecedentes de revascularización, cuya anatomía coronaria estaba intervenida.

Para analizar el impacto del SM y la DM de manera independiente, se dividió la muestra en tres grupos que se compararon entre sí:

-Pacientes sin SM, ni DM

-Pacientes con SM, pero sin DM

-Pacientes portadores de DM

Marcadores Bioquímicos: Los niveles de glicemia fueron medidos según el método de hexoquinasa glucosa 6-fosfato, el perfil lipídico por técnica enzimática y la glicohemoglobina por inmunoensayo.

Definiciones: Se consideró insuficiencia renal crónica (IRC) a la existencia de un Clearance de Creatinina $<60 \mathrm{ml} / 1,73 \mathrm{mt}$, calculado por la fórmula de Cockcroft. El diagnóstico de Dislipidemia se hizo de acuerdo a criterios ATP IIIa ${ }^{5}$. Para el diagnóstico de SM se utilizó criterios ATP IIIa, con los cortes de cintura utilizados en la Encuesta Nacional de Salud 2010 del Ministerio de Salud. Es decir 3 o más de 5 criterios: Circunferencia cintura elevada (>88 hombres y $>83 \mathrm{~cm}$ mujeres), Colesterol HDL $<40$ $\mathrm{mg} / \mathrm{dl}$ en hombres o $<50 \mathrm{mg} / \mathrm{dl}$ en mujeres, Triglicéridos $>$ 150 mg/dl, Presión Arterial > 130 / 85 mmHg y Glicemia $>100 \mathrm{mg} / \mathrm{dl}^{19}$.

Se catalogó como diabético a todo paciente con diagnóstico previo o con glicemia de ayuno mayor a $126 \mathrm{mg} / \mathrm{dl}$, criterio utilizado en la Encuesta Nacional de Salud. Se consideró como portador de CC a todo paciente con estenosis $>50 \%$ en cualquier arteria coronaria. La severidad de la anatomía coronaria fue medida a través del puntaje 
de Gensini, que asigna puntaje por el porcentaje de estenosis de la lesión y su importancia topográfica en el árbol coronario $^{20}$.

Análisis estadístico: Para establecer la magnitud de la aterosclerosis entre los distintos grupos, se comparó el puntaje de Gensini utilizando análisis de varianza (Barlett) con comparación múltiple de Scheffe y prueba no paramétrica de Kruskal-Wallis en aquellos grupos con varianzas distintas. Se utilizó prueba de t para poblaciones independientes. Se utilizó $\mathrm{Chi}^{2}$ para comparar variables discretas. La correlación entre puntaje de Gensini con IMC, colesterol, hemoglobina glicosilada y creatinina se realizó mediante regresión lineal. En todas las pruebas, se consideró una diferencia estadísticamente significativa, si el valor de $\mathrm{p}$ era inferior a 0.05 . Se utilizó software Stata $12 ®$ para Windows.

El protocolo fue aprobado por el Comité Ético Científico del Servicio de Salud Araucanía Sur.

\section{Resultados:}

Características de la población: Un total de 583 pacientes consecutivos fueron estudiados con coronariografía invasiva. De éstos, se excluyeron 32 pacientes que estaban cursando con SCA, 43 pacientes con cirugía de revascularización miocárdica y 76 pacientes con angioplastía percutánea previa. Además, 19 pacientes fueron excluidos por no tener datos completos, finalizando con una muestra de 413 pacientes.

El promedio de edad fue de 63,84 años y sexo masculino en $58,1 \%$. Las características de la población estudiada se muestran en la Tabla 1.

\begin{tabular}{|l|l|}
\hline \multicolumn{2}{|c|}{ Tabla 1. Características generales de la } \\
población estudiada \\
\hline Total pacientes & 413 pacientes \\
\hline Edad & $63,84+11,17$ \\
\hline Sexo Masculino & $58,1 \%$ \\
\hline Enfermedad Coronaria & $52,7 \%$ \\
\hline Factores de Riesgo Tradicionales & \\
\hline HTA & $79,1 \%$ \\
\hline Tabaquismo & $17,9 \%$ \\
\hline Dislipidemia & $87,3 \%$ \\
\hline Insuficiencia renal crónica & $20,2 \%$ \\
\hline Estado Nutricional según IMC & \\
\hline Normal < 25 IMC & $9,3 \%$ \\
\hline Sobrepeso 25 - 30 IMC & $27,2 \%$ \\
\hline Obesos > 30 IMC & $63 \%$ \\
\hline
\end{tabular}

Resultados de coronariografía y score de Gensini: La angiografía coronaria demostró CC en $52,7 \%$ de los pacientes y, de ellos, el 23,5\% tuvo enfermedad de 1 vaso, $13,9 \%$ de 2 vasos, y $15,3 \%$ de 3 vasos. El Score de Gensini promedio en la muestra fue $23.9 \pm 34,5$ con valores entre 0 y 146. La coronariografía fue normal $(G e n s i n i=0)$ en un $44,1 \%$ de los pacientes.

Impacto de la enfermedad metabólica: Al dividir la muestra total en los tres grupos definidos previamente, $16,7 \%(\mathrm{n}=69)$ no demostraron enfermedad metabólica, 38,2\% (n=158) presentaban SM (no diabéticos) y $45 \%(\mathrm{n}=186)$ de los pacientes eran diabéticos.

Hubo similar porcentaje de varones en los 3 grupos $(58,8 \%, 54,4 \%$ y $56,2 \%$, respectivamente, $\mathrm{p}=0,1)$. Con respecto a la edad, el grupo de pacientes diabéticos fue mayor (66 años vs 64,7 en aquellos con SM y 64,7 en los sin alteración metabólica, p 0,0132). Esta diferencia es significativa entre el grupo DM y el grupo SM (p 0,007), no existiendo diferencias entre pacientes con SM y sin alteraciones ( $\mathrm{p} 0,307)$.

Respecto al peso, existieron diferencias significativas entre SM y sin alteraciones metabólicas $(74,9 \pm 12,7$ vs 69,54 $\pm 12,56 \mathrm{Kg}$, respectivamente, $\mathrm{p} 0,014)$. No existió diferencias entre pacientes con SM y aquellos con DM.

El IMC fue menor en pacientes sin alteraciones metabólicas comparado con pacientes con SM y DM $(26,5 \pm 4,66$ vs $28,4 \pm 4,95$ y $28,1 \pm 4,22$, respectivamente p 0,0212 ).

En relación a la prevalencia de Hipertensión Arterial (HA) entre los distintos grupos, se observó un mayor porcentaje de este factor de riesgo en aquellos pacientes diabéticos ( $88,6 \%$ vs 74,5 y $75 \%$, p 0,002$)$.

Hubo diferencias significativas en cuanto al porcentaje de pacientes con IRC, siendo mayor su prevalencia en pacientes diabéticos en comparación con pacientes con SM y sin alteraciones $(27,5 \%$ vs $15,4 \%$ y $16,6 \%$ p 0,034$)$. En la Tabla 2 se demuestran las características de los subgrupos estudiados.

Relación con la presencia de Cardiopatía coronaria: se observaron claras diferencias con respecto a la $\mathrm{CC}$, siendo su prevalencia mayor en pacientes con SM y DM versus los pacientes sin alteraciones metabólicas $(62,2 \%$ y $54,2 \%$ vs $33,9 \%$, p 0,001). Asimismo, la severidad de la CC en estos pacientes fue mayor, existiendo un puntaje de Gensini mayor en pacientes diabéticos, o con SM, comparado con el grupo sin alteración metabólica; 29,6 $\pm 38,98,22,04 \pm 31,36$ y $18 \pm 32,7$, respectivamente $(\mathrm{p} 0,0082)$ (Tabla 3 ).

Hubo una correlación del índice cintura/cadera, tanto con 


\begin{tabular}{|l|r|r|r|r|}
\multicolumn{5}{|c}{ Tabla 2. Características demográficas y clínicas de los } \\
grupos estudiados \\
\hline
\end{tabular}

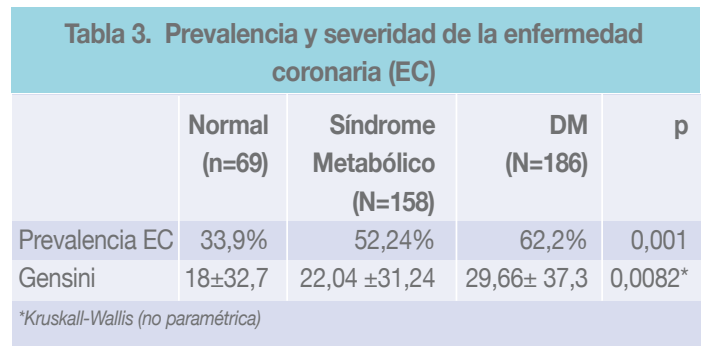

el puntaje de Gensini como con la prevalencia de CC. El quintil más bajo de este índice presentó una prevalencia de CC menor comparado con el quintil más alto ( $34 \%$ vs $56 \% \mathrm{p}=0,001$ ) (Tabla 4). Comparando estos dos quintiles el odds ratio para la prevalencia de enfermedad coronaria fue $2,00((1,04-3,85)$ p 0,0246$)$. Esta relación no se obser-

\begin{tabular}{|l|r|r|}
\hline \multicolumn{3}{|c|}{ Tabla 4. Prevalencia de enfermedad coronaria y } \\
medidas antropométricas
\end{tabular}

vó respecto del IMC.

La regresión lineal mostró una correlación débil del score de Gensini con la edad ( $\mathrm{r} 20,11 \mathrm{p}=0,0223)$, índice cintura-cadera $(\mathrm{r} 2=0,14 \mathrm{p}=0.0046)$, hemoglobina glicosilada (r2 0,15 $\mathrm{p}=0,02)$ e inversamente proporcional con el CHDL $(\mathrm{r} 2=-0,15 \mathrm{p}=0,011)$. No hubo asociación al comparar peso, IMC, clearance de creatinina, colesterol total, CLDL, ni glicemia con el puntaje Gensini (Figura 1).

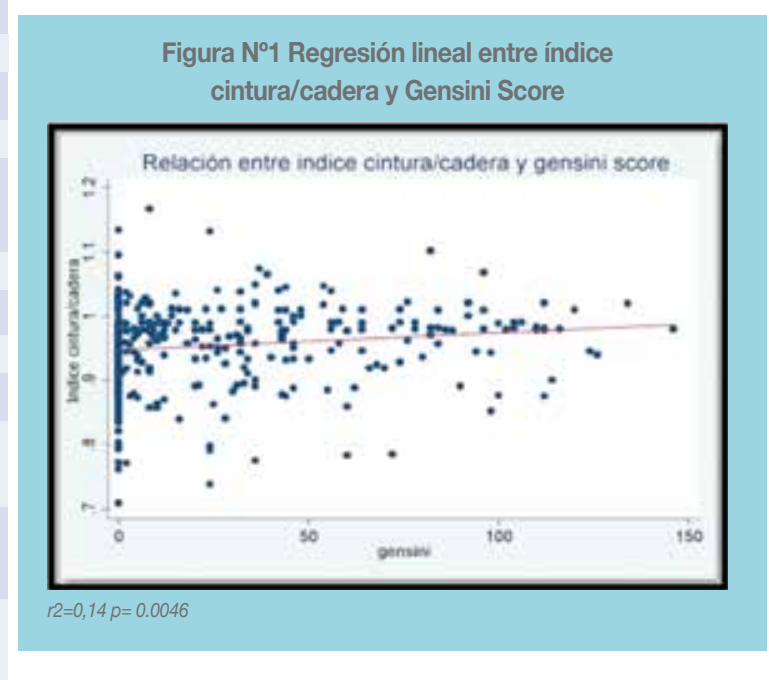

\section{Discusión:}

Este estudio demuestra fundamentalmente tres hechos:

1. La existencia de enfermedad metabólica confiere un mayor riesgo de enfermedad coronaria.

2. Dicho riesgo es mayor en pacientes portadores de DM comparado con los con SM (sin DM).

3. La severidad de la CC medida por índice de Gensini es igualmente mayor en pacientes con DM.

En nuestra muestra, tanto la DM como el SM se asociaron a mayor prevalencia y severidad de CC, en comparación con pacientes sin enfermedad metabólica.

El factor determinante de prevalencia y severidad de la enfermedad coronaria en pacientes con DM es un hecho conocido $^{6} \mathrm{y}$ el futuro de estos pacientes está asociado a un pronóstico más ominoso ${ }^{21}$. En nuestra muestra la prevalencia de $\mathrm{CC}$ en pacientes diabéticos fue de $62,2 \%$. Esta alta cifra se explica dado que la población incluida en nuestro estudio tenía algún grado de sospecha de enfermedad coronaria debido a que el diagnóstico fue realizado por coronariografía y no por la clínica.

Existen reportes que asocian mayor ateroesclerosis a medida que aumenta la hemoglobina glicosilada y la duración de la DM. En un estudio, donde se realizó coronariografía a 110 pacientes con (SCA), 78 de ellos diabéticos, encon- 
traron relación directa entre puntaje de Gensini y Hemoglobina glicosilada $(\mathrm{r}=0.427, \mathrm{p}=0.001)$ [22]. Hallazgo similar a lo reportado en nuestro estudio, si bien la correlación en nuestra muestra fue menor $(r=0,15 \mathrm{p}=0,02)$.

También existe evidencia que relaciona mayor severidad de CC con la variabilidad glicémica. En un ensayo que estudió 344 pacientes diabéticos sometidos a coronariografía, se encontró relación directa entre puntaje de Gensini y amplitud promedio de las excursiones glicémicas $(\mathrm{r}=0.277, \mathrm{p}<0.001)^{23}$.

La literatura es controversial con respecto al real impacto del SM. En un estudio realizado en 632 pacientes sometidos a coronariografía no se encontraron diferencias entre sujetos con o sin SM y score de Gensini ${ }^{24}$ : Por otro lado no se ha asociado una mayor prevalencia de obstrucción arterial periférica en pacientes con $\mathrm{SM}^{25}$. En otro estudio, donde se sometieron 1003 pacientes a coronariografía, los pacientes con SM presentaron un puntaje de Gensini mayor en comparación a pacientes sin este factor de riesgo (25 vs $18 \mathrm{p}<0,001)^{26}$. Este último hecho es concordante con nuestros resultados.

En una evaluación del impacto del SM durante el seguimiento de 480 pacientes consecutivos con SCA, se reportó un aumento en la mortalidad total en el grupo con SM, comparado al grupo sin $\mathrm{SM}^{27}$. Por otro lado el estudio DECODE, que estudió pacientes con SM no diabéticos, demostró en ellos mayor riesgo de muerte por enfermedad cardiovascular OR 2,26 (95\% CI, 1.61-3.17) para hombres y $2,78\left(95 \%\right.$ CI, 1.57-4.94) para mujeres ${ }^{28}$.

La definición de SM es distinta en las diversas recomendaciones, algunas de las cuales obligan a medir resistencia a la insulina. Escogimos la de ATP-III, por ser más simple y utilizar variables más fáciles de obtener. Asimismo, este criterio es el más ampliamente difundido y utilizado en la Encuesta Nacional de Salud de nuestro país ${ }^{19}$. Así mismo adoptamos catalogar a los pacientes como DM utilizando criterios de la ENS por ser una definición operativa, y dado la poca factibilidad de realizar test tolerancia a la glucosa a un alto número de pacientes.

La severidad de la enfermedad coronaria se midió con el score de Gensini por ser un índice validado, relativamente simple y de buena reproducibilidad ${ }^{23}$. No se utilizó score de SYNTAX, dado que éste se diseñó para evaluar la complejidad de las lesiones coronarias y decidir conducta terapéutica entre cirugía y angioplastía percutánea, más que evaluar la severidad en cuanto a la carga aterosclerótica en la coronariografía.

\section{Otros resultados:}

La mayor prevalencia de IRC en pacientes diabéticos en la muestra es concordante con lo descrito en la literatura ${ }^{29}$.

Nuestros pacientes tenían igualmente una cantidad importante de otros factores de riesgo cardiovascular como dislipidemias, HTA e índice cintura cadera alterado, siendo esto más acentuado en los pacientes con DM. Existió una asociación positiva entre el índice cintura-cadera y la carga de aterotrombosis medida con el puntaje de Gensini, con un coeficiente de relación $\mathrm{r} 2=14 \mathrm{p}=0,004$. El quintil más bajo de este índice presentó una prevalencia de enfermedad coronaria mucho menor comparado con el quintil más alto $(35 \%$ vs $50 \%$ p $=0,001)$. Esta correlación no se observó al comparar con el IMC. Eso es compatible con lo publicado, que indica que la obesidad por sí sola no siempre incrementa el riesgo cardiovascular ${ }^{30}$. En el estudio INTERHEART, el índice cintura-cadera fue la medida antropométrica más potente relacionada con cardiopatía coronaria, con aumento del odds ratio para infarto al miocardio a medida que lo hace el quintil (OR $=1.15$ para el 2 do quintil, 1.39 para el 3er quintil, 1.9 para el 4to quintil y 2.52 para el 5to quintil; $\mathrm{P}<.0001) .{ }^{31}$ Los individuos con predominio de la grasa subcutánea, con distribución ginecoide presentan un riesgo cardiovascular, para un índice de masa corporal (IMC) determinado, menor que aquellos con un patrón androide, propio de la obesidad visceral. Actualmente, se considera la grasa visceral como un órgano endocrino dinámico, muy activo en secreción de hormonas y adipoquinas ${ }^{32-33}$. Por este motivo, el IMC no predice de forma aislada el aumento del riesgo cardiovascular.

Como conclusión, esta muestra sugiere que tanto el SM como la DM son predictores de mayor enfermedad aterosclerótica. No hay estudios que hayan analizado la carga aterosclerótica coronaria en pacientes con SM en nuestro país, lo que constituye un hallazgo importante. Asimismo, el concepto de SM es útil ya que se ajusta al perfil de muchos pacientes que acuden a la atención primaria en la actualidad, lo que puede incentivar a todos los profesionales de Salud a tratar de controlar de forma más estricta los factores de riesgo y modificar los estilos de vida poco saludables. 


\section{Referencias:}

1. MINSAL. Departamento de estadísticas e información de Salud. 2010.

2. COUTINHO M, GERSTEIN HC, WANG Y, YUSUF S., The relationship between glucose and incident cardiovascular events. A metaregression analysis of published data from 20 studies of 95,783 individuals followed for 12.4 years. Diabetes Care, 1999; 22: 233-40.

3. REAVEN GM. Insulin resistance: the link between obesity and cardiovascular disease. Med Clin North Am, 2011; 95: 875-92.

4. YUSUF S, HAWKEN S, OUNPUU S, DANS T, AVEZUM A, LANAS F, et al. Effect of potentially modifiable risk factors associated with myocardial infarction in 52 countries (the INTERHEART study): case-control study. Lancet, 2004; 364 : 937-52.

5. Third Report of the National Cholesterol Education Program (NCEP) Expert Panel on Detection, Evaluation, and Treatment of High Blood Cholesterol in Adults (Adult Treatment Panel III) final report. Circulation, 2002; 106: 3143-421.

6. NATALI A, VICHI S, LANDI P, SEVERI S, L'ABBATE A, FERRANNINI E. Coronary atherosclerosis in Type II diabetes: angiographic findings and clinical outcome. Diabetologia, 2000; 43: 632-41.

7. ANAND DV, LIM E, LAHIRI A, BAX JJ. The role of non-invasive imaging in the risk stratification of asymptomatic diabetic subjects. Eur Heart J, 2006; 27: 905-12.

8. HU FB, STAMPFER MJ, HAFFNER SM, SOLOMON CG, WILLETT WC, MANSON JE, Elevated risk of cardiovascular disease prior to clinical diagnosis of type 2 diabetes. Diabetes Care, 2002; 25: 1129-34.

9. REAVEN G.M. Banting lecture 1988. Role of insulin resistance in human disease. Diabetes, 1988; 37: 1595-607.

10. FORD ES. Risks for all-cause mortality, cardiovascular di- sease, and diabetes associated with the metabolic syndrome: a summary of the evidence. Diabetes Care, 2005; 28: 1769-78.

11. GALASSI A, K REYNOLDS, HE J, Metabolic syndrome and risk of cardiovascular disease: a meta-analysis. Am J Med, 2006; 119: 812-9.

12. GAMI AS, WITT BJ, HOWARD DE, ERWIN PJ, GAMI LA, SOMERS VK, et al., Metabolic syndrome and risk of incident cardiovascular events and death: a systematic review and meta-analysis of longitudinal studies. J Am Coll Cardiol, 2007; 49: 403-14.

13. LAKKA HM, LAAKSONEN DE, LAKKA TA, NISKANEN LK, KUMPUSALO E, TUOMILEHTO J, et al. THe metabolic syndrome and total and cardiovascular disease mortality in middle-aged men. JAMA, 2002; 288: 2709-2716.

14. SOLYMOSS BC, BOURASSA MG, LESPÉRANCE J, LEVESQUE S, MARCIL M, VARGA S, et al. Incidence and clinical characteristics of the metabolic syndrome in patients with coronary artery disease. Coron Artery Dis, 2003; 14: 207-12.

15. SUNDSTRÖM J, VALLHAGEN E, RISÉRUS U, BYBERG L, ZETHELIUS B, BERNE C, et al. Risk associated with the metabolic syndrome versus the sum of its individual components. Diabetes Care, 2006; 29: 1673-4.

16. REAVEN GM, The metabolic syndrome: requiescat in pace. Clin Chem, 2005; 51: 931-8.

17. ALBERTI KG , ZIMMET PZ. Definition, diagnosis and classification of diabetes mellitus and its complications. Part 1: diagnosis and classification of diabetes mellitus provisional report of a WHO consultation. Diabet Med, 1998; 15: 539-53.

18. ALBERTI KG , ZIMMET PZ, SHAW J, Metabolic syndrome-a new world-wide definition. A Consensus Statement from the International Diabetes Federation. Diabetic Medicine, 2006; 23: 469-480. 
19. MINSAL, Encuesta Nacional de Salud ENS 2009-2010. 2010.

20. GENSINI G. A more meaningful scoring system for determining the severity of coronary heart disease. Am J Cardiol, 1983; 51: 606 .

21. STEIN B, WEINTRAUB WS, GEBHART SP, COHEN-BERNSTEIN CL, GROSSWALD R, et al. Influence of diabetes mellitus on early and late outcome after percutaneous transluminal coronary angioplasty. Circulation, 1995; 91: 979-89.

22. SALEEM T, MOHAMMAD KH, ABDEL-FATTAH MM, ABBASI AH. Association of glycosylated haemoglobin level and diabetes mellitus duration with the severity of coronary artery disease. Diab Vasc Dis Res, 2008; 5: 184-9.

23. SU G, MI S, TAO H, LI Z, YANG H, ZHENG H, et al. Association of glycemic variability and the presence and severity of coronary artery disease in patients with type 2 diabetes. Cardiovasc Diabetol, 2011; 10: 19.

24. KIM JY, MUN HS, LEE BK, YOON SB, CHOI EY, MIN $\mathrm{PK}, \mathrm{ET}$ AL. Impact of metabolic syndrome and its individual components on the presence and severity of angiographic coronary artery disease. Yonsei Med J, 2010; 51: 676-82.

25. Maksimovic M, Vlajinac H, Radak D, Marinkovic J, Jorga J. Relationship between peripheral arterial disease and metabolic syndrome. Angiology, 2009; 60: 546-53.

26. YAVUZ B, KABAKCI G, AKSOY H, TULUMEN E, DEVECI OS, AYTEMIR K, et al., Determining the relationship between metabolic syndrome score and angiographic severity of coronary artery disease. Int J Clin Pract, 2008; 62: 717-22.

27. BOULON C, LAFITTE M, RICHEBOEUF V, PAVIOT B, PRADEAU V, COSTE P, et al., Prevalence of metabolic syndrome after acute coronary syndrome and its prognostic significance. Am J Cardiol, 2006; 98: 1429-34.

28. HU G, QIAO Q, TUOMILEHTO J, BALKAU B, BORCH-JOHNSEN K, PYORALA K. PRevalence of the metabolic syndrome and its relation to all-cause and cardiovascular mortality in nondiabetic european men and women. Archives of Internal Medicine, 2004; 164: 1066-1076.

29. MCCLELLAN W. Epidemiology and risk factors for chronic kidney disease. Med Clin North Am, 2005; 89: 419-45.

30. SCHULTE H, CULLEN P, ASSMANN G. Obesity, mortality and cardiovascular disease in the Munster Heart Study (PROCAM). Atherosclerosis, 1999; 144: 199-209.

31. YUSUF S, HAWKEN S, OUNPUU S, BAUTISTA L, FRANZOSI MG, COMMERFORD P, ET AL. Obesity and the risk of myocardial infarction in 27,000 participants from 52 countries: a case-control study. Lancet, 2005; 366: 1640-9.

32. BAUDRAND BR, ARTEAGA UE, MORENO G.M. Adipose tissue as an endocrine modulator: hormonal changes associated with obesity. Rev Med Chil, 2010; 138: 1294-301.

33. Rocha V, Libby P. The multiple facets of the fat tissue. Thyroid, 2008; 18: 175-83. 